\title{
New Concept of Factorials and Combinatorial Numbers and its Consequences for Algebra and Analysis
}

\author{
Mohamed E. Hassani ${ }^{1}$ \\ Institute for Fundamental Research \\ BP.197, CTR, GHARDAIA 47800, ALGERIA
}

(7 June 2021)

\begin{abstract}
In this article, the usual factorials and binomial coefficients have been generalized and extended to the negative integers. Basing on this generalization and extension, a new kind of polynomials has been proposed, which led directly to the non-classical hypergeometric orthogonal polynomials and the non-classical second-order hypergeometric linear DEs. The resulting polynomials can be used in non-relativistic and relativistic QM, particularly, in the case of the Schrödinger equation, and Dirac equations for an electron in a Coulomb potential field.
\end{abstract}

Keyword: factorials, binomial coefficients, combinatorial numbers, non-classical hypergeometric orthogonal polynomials, non-classical second-order hypergeometric linear DEs

$\operatorname{MSC}(\mathbf{2 0 2 0}): 05 \mathrm{~A} 10 ; 33 \mathrm{C} 45 ; 34 \mathrm{~B} 30$

\section{Introduction}

Usually, the factorial of a positive integer $n$, denoted by $n$ !, is defined as the product of all positive integers less than or equal to $n: n !=n \times(n-1) \times(n-2) \times(n-3) \times \cdots \times 3 \times 2 \times 1$. The value of 0 ! is conventionally equal to 1 . However, the factorials of negative integers cannot be computed, since for $n$ $=0$, the recurrence relation $(n-1) !=n ! / n$ implies division by zero, and also due to the fact that the usual binomial coefficient $C_{n}^{k} \equiv\left(\begin{array}{l}n \\ k\end{array}\right)=n ! / k !(n-k)$ ! is always equal to a positive integer.

The factorials are mostly encountered in many areas of mathematics, notably in combinatorics, probability theory, number theory, statistics, algebra, and mathematical analysis. Actually, their most basic use counts the possible distinct sequences the 'permutations' of $n$ distinct objects: there are $n$ !. The factorials can also be extended to real numbers while retaining its most important properties. This involves using gamma function to define $\Gamma(x+1)=x$ !. But, as it is abovementioned, this extension does not work when $x$ is a negative integer.

The factorials have a long and fascinating history $[1,2]$. They were used to count permutations at least as early as the 12th century, by Indian scholars [3]. In 1677, Fabian Stedman described factorials as applied to change ringing, a musical art involving the ringing of many tuned bells [4]. In 1808, the French mathematician Christian Kramp introduced the notation $n$ ! [5].

Concerning the gamma function and the extension of factorials to real negative numbers, many famous mathematicians worked on this topic, particularly Euler, Bernoulli (Daniel) Goldbach and De Moivre [6, 7].

\footnotetext{
${ }^{1}$ E-mail:hassani641@gmail.com
} 
As a matter of fact, the field of factorials has attracted many modern researchers whose goal was the generalization of factorials and/or the extension of gamma function $[8,9,10,11,12]$. However, the purpose and expectations of the present work are radically different from what was already published on the topic under discussion as we will see soon.

\section{Generalization of factorials to negative integers}

The usual factorials of positive integers can be generalized to negative integers as follows. We have

$$
n !=1 \times 2 \times 3 \times \cdots \times n, \quad n \in \mathbf{N}^{*} .
$$

It is quite clear that multiplying each integer on the RHS of (1) by the constant $\varepsilon= \pm 1$ is equivalent to multiply the LHS of (1) by $(\varepsilon)^{n}$ :

$$
(\varepsilon)^{n} n !=(\varepsilon 1) \times(\varepsilon 2) \times(\varepsilon 3) \times \cdots \times(\varepsilon n) .
$$

Putting $\varepsilon=-1$ in (2) yields

$$
(-1)^{n} n !=(-1) \times(-2) \times(-3) \times \cdots \times(-n) .
$$

Using the notation $(-n) !=(-1) \times(-2) \times(-3) \times \cdots \times(-n)$ to rewrite (3) in the following compact form

$$
(-n) !=(-1)^{n} n !
$$

As we can see, the relation (4) is, in fact, a special case of

$$
(\varepsilon n) !=(\varepsilon)^{n} n ! \text {. }
$$

We call the relation (4) 'factorials of negative integers', therefore, Eq.(5) may be understood as a generalization of factorials to negative integers and beyond. Now, return to (1) and rewrite it in the following form

$$
(n !)=(1 \times 2 \times 3 \times \cdots \times n),
$$

Multiplying the two sides of (6) by -1 to get

$$
-(n !)=-(1 \times 2 \times 3 \times \cdots \times n) .
$$

From (4) and (7), we arrive at the result: in general

$$
-(n !) \neq(-n) ! .
$$

Definition 1: we call a factorial number any expression of the form

1. $(-n) !=[-(n)] !=(-1)^{n} n$ !

2. $(-n+k) !=[-(n-k)] !=(-1)^{n-k}(n-k)$ !

3. $(-n-k) !=[-(n+k)] !=(-1)^{n+k}(n+k)$ ! 
Result 1: from (1), (4) and (5) we get

$$
\varepsilon^{n} n !=\left\{\begin{array}{ll}
n ! & \text { if } \varepsilon=1 \\
(-n) ! & \text { if } \varepsilon=-1
\end{array},\right.
$$

where $n \in \mathbf{Z}_{+} \equiv \mathbf{N}$ and $-n \in \mathbf{Z}_{-}$.

\section{Generalization of binomial coefficients to negative integers}

The previous generalization of factorials to negative integers allows us to generalize the binomial coefficients to negative integers along these lines. We have

$$
C_{n}^{k} \equiv\left(\begin{array}{l}
n \\
k
\end{array}\right)=\frac{n !}{k !(n-k) !}=\frac{(n) !}{(k) !(n-k) !}
$$

Replacing $n$ by $-n$ in both sides of (10) to get

$$
C_{-n}^{k}=\frac{(-n) !}{(k) !(-n-k) !}
$$

Taking into account Eq.(4) and definition (1), the expression (11) becomes

$$
C_{-n}^{k}=(-1)^{k} \frac{n !}{k !(n+k) !} .
$$

Again, replacing $k$ by $-k$ in both sides of (10) yields

$$
C_{n}^{-k}=(-1)^{k} \frac{n !}{k !(n+k) !}
$$

Result 2: from (12) and (13), we get

$$
C_{-n}^{k}=C_{n}^{-k}
$$

Finally, replacing $n$ and $k$ by $-n$ and $-k$, respectively, in both sides of (10) to obtain

$$
C_{-n}^{-k}=\frac{n !}{k !(n-k) !} .
$$

Result 3: comparing (9) and (15) yields

$$
C_{-n}^{-k}=C_{n}^{k}
$$

Definition 2: we call a combinatorial number any expression of the form (12), (13) and (15).

As we will see, the usual factorials of positive integers are, actually, a special case of Eq.(5), that is to say, when $\varepsilon=1$. Furthermore, the usual binomial coefficients are generalized to negative integers via the formulae (12), (13) and (15). To clarify all that, some usual factorials of positive integers, factorial numbers (factorials of negative integers), usual binomial coefficients and combinatorial numbers are, respectively, listed in Tables 1 and 2. 
4

\begin{tabular}{rr|rr}
\hline \hline$n$ & $n !$ & $-n$ & $(-n) !=(-1)^{n} n !$ \\
\hline & & & \\
0 & 1 & 0 & 1 \\
1 & 1 & -1 & -1 \\
2 & 2 & -2 & 2 \\
3 & 6 & -3 & -6 \\
4 & 24 & -4 & 24 \\
5 & 120 & -5 & -120 \\
6 & 720 & -6 & 720 \\
7 & 5040 & -7 & -5040 \\
8 & 40320 & -8 & 40320 \\
9 & 362880 & -9 & -362880 \\
10 & 3628800 & -10 & 3628800 \\
\hline
\end{tabular}

Table 1: some usual factorials of positive integers and some factorial numbers

\begin{tabular}{ccc|ccc}
\hline \hline$n$ & $k$ & $C_{n}^{k}=n ! /(n-k) !$ & $-n$ & $k$ & $C_{-n}^{k}=(-1)^{k} n ! /(n+k) !$ \\
\hline 1 & 0 & 1 & -1 & 0 & 1 \\
2 & 1 & 2 & -2 & 1 & $-1 / 3$ \\
3 & 2 & 3 & -3 & 2 & $1 / 40$ \\
4 & 3 & 4 & -4 & 3 & $-1 / 1225$ \\
5 & 4 & 5 & -5 & 4 & $1 / 725776$ \\
6 & 5 & 6 & -6 & 5 & $-1 / 6652800$ \\
\hline \hline
\end{tabular}

Table 2: some usual binomial coefficients and combinatorial numbers

\subsection{Some generalized combinatorial formulae}

We deduce from definitions 1 and 2 the following interesting formulae which can be useful later.
I. $C_{-n+k}^{k}=(-1)^{k} \frac{(n-k) !}{n ! k !}$
II. $\frac{C_{-n}^{-k}}{C_{n}^{-k}}=\frac{C_{n}^{k}}{C_{-n}^{k}}=(-1)^{k} \frac{(n+k) !}{(n-k) !}$
III. $C_{-n+k}^{k} \cdot C_{-n}^{-k}=C_{-n+k}^{k} \cdot C_{n}^{k}=\frac{(-1)^{k}}{(k !)^{2}}$
IV. $C_{-n}^{-k} \cdot C_{n}^{k}=\left[\frac{n !}{k !(n-k) !}\right]^{2}$ 

V. $C_{-n}^{k} \cdot C_{n}^{-k}=\left[\frac{n !}{k !(n+k) !}\right]^{2}$
VI. $C_{-n}^{-k} \cdot C_{-n}^{k}=C_{n}^{k} \cdot C_{n}^{-k}=\frac{(-1)^{k}}{(n+k) !(n+k) !}\left[\frac{n !}{k !}\right]^{2}$

\subsection{Application of the generalized combinatorial numbers}

The concept of the generalized combinatorial numbers as a generalization of the usual binomial coefficients allows us to introduce some new kind of polynomials in which the generalized combinatorial numbers defined by formulae 12,13,15 and I-VI, playing the role of coefficients. At present, let us begin with the polynomial $H_{n}\left(x^{-1}, y^{-1}\right)$ of degree $n$ in $x^{-1}$ and $y^{-1}$ :

$$
H_{n}\left(x^{-1}, y^{-1}\right)=\sum_{k=0}^{n} \frac{a_{n, k}}{x^{n-k} y^{k}},
$$

where $x, y \in \mathbf{R}^{*}$ or $\mathbf{C}^{*} ; n, k \in \mathbf{N}$ and the coefficients $a_{n, k}$ are the generalized binomial coefficients (generalized combinatorial numbers). In this sense, $a_{n, k} \in \mathcal{C}$ where $\mathcal{C}=\{12,13,15$, I, II, III, IV, V, $\mathrm{VI}$ \} is the set of the generalized binomial coefficients defined by the formulae 12, 13, 15 and I-VI. Furthermore, in order to understand correctly the role and importance of $a_{n, k}$ we make use of (17) to define the following polynomials:

$$
\begin{gathered}
a_{n, k} \in \mathscr{C} \mid a_{n, k}=C_{n}^{-k} ; H_{n}\left(x^{-1}, y^{-1}\right) \equiv P_{n}\left(x^{-1}, y^{-1}\right)=\sum_{k=0}^{n} C_{n}^{-k} \frac{1}{x^{n-k} y^{k}} \\
a_{n, k} \in \mathscr{C} \mid a_{n, k}=C_{-n}^{-k} ; H_{n}\left(x^{-1}, y^{-1}\right) \equiv Q_{n}\left(x^{-1}, y^{-1}\right)=\sum_{k=0}^{n} C_{-n}^{-k} \frac{1}{x^{n-k} y^{k}} .
\end{gathered}
$$

First, we begin with (18) which can be written in explicit form as follows :

$$
\begin{aligned}
P_{n}\left(x^{-1}, y^{-1}\right) & =\sum_{k=0}^{n}(-1)^{n} \frac{n !}{k !(n+k) !} \frac{1}{x^{n-k}} \frac{1}{y^{k}} \\
& =\frac{1}{x^{n}}-\frac{n !}{1 !(n+1) !} \frac{1}{x^{n-1}} \frac{1}{y^{1}}+\frac{n !}{2 !(n+2) !} \frac{1}{x^{n-2}} \frac{1}{y^{2}}-\frac{n !}{3 !(n+3) !} \frac{1}{x^{n-3}} \frac{1}{y^{3}}+\cdots+\frac{(-1)^{n}}{(2 n) !} \frac{1}{y^{n}} .
\end{aligned}
$$

The first few P-polynomials

$$
\begin{aligned}
& P_{0}\left(x^{-1}, y^{-1}\right)=1 \\
& P_{1}\left(x^{-1}, y^{-1}\right)=x^{-1}-\frac{1}{2} y^{-1} \\
& P_{2}\left(x^{-1}, y^{-1}\right)=x^{-2}-\frac{1}{3} x^{-1} y^{-1}+\frac{1}{24} y^{-2} \\
& P_{3}\left(x^{-1}, y^{-1}\right)=x^{-3}-\frac{1}{4} x^{-2} y^{-1}+\frac{1}{40} x^{-1} y^{-2}-\frac{1}{720} y^{-3}, \\
& P_{4}\left(x^{-1}, y^{-1}\right)=x^{-4}-\frac{1}{5} x^{-3} y^{-1}+\frac{1}{60} x^{-2} y^{-2}-\frac{1}{1260} x^{-1} y^{-3}+\frac{1}{40320} y^{-4},
\end{aligned}
$$


6

$P_{5}\left(x^{-1}, y^{-1}\right)=x^{-5}-\frac{1}{6} x^{-4} y^{-1}+\frac{1}{84} x^{-3} y^{-2}-\frac{1}{2016} x^{-2} y^{-3}+\frac{1}{72576} x^{-1} y^{-4}-\frac{1}{39916800} y^{-5}$.

Derivative formulae for P-polynomial

$m^{\text {th }}$-order partial derivative of $P_{n}\left(x^{-1}, y^{-1}\right)$ w.r.t $x^{-1}, x, y^{-1}$ and $y$, respectively.

$$
\begin{aligned}
& \left(x^{-1}\right)^{m} \frac{\partial^{m} P_{n}}{\partial\left(x^{-1}\right)^{m}}=\sum_{k=0}^{n} C_{n}^{-k} \frac{(n-k)_{(m)}}{x^{n-k} y^{k}}, \\
& x^{m} \frac{\partial^{m} P_{n}}{\partial x^{m}}=(-1)^{m} \sum_{k=0}^{n} C_{n}^{-k} \frac{(n-k)^{(m)}}{x^{n-k} y^{k}}, \\
& \left(y^{-1}\right)^{m} \frac{\partial^{m} P_{n}}{\partial\left(y^{-1}\right)^{m}}=\sum_{k=0}^{n} C_{n}^{-k} \frac{k_{(m)}}{x^{n-k} y^{k}}, \\
& y^{m} \frac{\partial^{m} P_{n}}{\partial y^{m}}=(-1)^{m} \sum_{k=0}^{n} C_{n}^{-k} \frac{k^{(m)}}{x^{n-k} y^{k}},
\end{aligned}
$$

where $(n-k)_{(m)}, k_{(m)}$ are the falling factorials and $(n-k)^{(m)}, k^{(m)}$ are the rising factorials.

\section{Characteristic values}

$P_{0}\left(x^{-1}, y^{-1}\right)=1$

$P_{n}(1,1)=\sum_{k=0}^{n} C_{n}^{-k}$

$P_{n}(\infty, \infty)=1$

$\left.P_{n}\left(x^{-1}, y^{-1}\right)\right|_{(x, y) \rightarrow(0,0)}=\infty$.

Parity

$P_{n}\left(-x^{-1},-y^{-1}\right)=(-1)^{n} P_{n}\left(x^{-1}, y^{-1}\right)$.

Property

Now, supposing $y$ is fixed thus by putting $\alpha=y^{-1}$ and $z=y^{-1}$ we get, after substitution in (20), the polynomial $P_{n}(z, \alpha)$ of order $\alpha$ and degree $n$ in $z$ :

$$
P_{n}(z, \alpha)=\sum_{k=0}^{n} C_{n}^{-k} z^{n-k}=n !\left[\frac{z^{n}}{n !}-\frac{\alpha^{1}}{1 !(n+1) !} z^{n-1}+\frac{\alpha^{2}}{2 !(n+2) !} z^{n-2}-\frac{\alpha^{3}}{3 !(n+3) !} z^{n-3}+\cdots+(-1)^{n} \frac{\alpha^{n}}{n !(2 n) !}\right] .
$$

The polynomial (26) satisfies the second-order self-adjoint DE

$$
\frac{d}{d z}\left(z^{2} \frac{d u}{d z}\right)-\lambda u=z^{\gamma} f_{n}(z, \alpha)
$$


for the interesting special case when $\lambda=n(n+1)$ and $\gamma=n$, where

$$
f_{n}(z, \alpha)=\sum_{k=0}^{n} C_{k n}\left(\frac{\alpha}{z}\right)^{k}, C_{k n}=(-1)^{k+1} \frac{k(2 n-k+1)}{k !(n+1)^{(k)}}
$$

\section{Non-classical hypergeometric orthogonal polynomials}

The well-known sets of orthogonal polynomials (OPs) of Jacobi polynomials $\left\{p_{n}^{(\alpha, \beta)}(x)\right\}_{n=0}^{\infty}$, Laguerre polynomials $\left\{L_{n}^{(a)}(x)\right\}_{n=0}^{\infty}$, Hermite polynomials $\left\{H_{n}(x)\right\}_{n=0}^{\infty}$, and Bessel polynomials $\left\{y_{n}^{(a)}(x)\right\}_{n=0}^{\infty}$ (including as an important special case the Legendre polynomials, Chebyshev polynomials, and Gegenbauer polynomials) are usually called classical (hypergeometric) OPs [16, 17, 18, 19] because they satisfy the well-known four classical hypergeometric linear DEs, namely:

Jacobi Eq.:

$$
\left(1-x^{2}\right) y^{\prime \prime}+[\beta-\alpha-(\alpha+\beta+2) x] y^{\prime}+n(n+\alpha+\beta+1) y=0,
$$

Laguerre Eq.:

$$
x y^{\prime \prime}+(a+1-x) y^{\prime}+n y=0,
$$

Hermite Eq.: $\quad y^{\prime \prime}-2 x y^{\prime}+2 n y=0$,

Bessel Eq.:

$$
x^{2} y^{\prime \prime}+(a x+b) y^{\prime}-n(n+a-1) y=0 \text {. }
$$

Also the classical OPs have the property that their derivatives form orthogonal systems.

In addition to the above mentioned classical OPs, there is a new class of OPs $\left\{\pi_{n}^{(\alpha, \beta)}(z)\right\}_{n=0}^{\infty}$ which do not belong to the classical sets of OPs and they are called non-classical (hypergeometric) OPs for the reason that besides their property of orthogonality, they satisfy a non-classical second-order hypergeometric linear DE of the form:

$$
A(z) u^{\prime \prime}+B(z, \alpha, \beta, n) u^{\prime}+\lambda_{n} u=0
$$

such that the coefficients of $u^{\prime \prime}$ and $u^{\prime}$ are quadratic and/or linear polynomials; $(\alpha, \beta)$ and $n$ are, respectively, bi-order and degree of the polynomial solutions of Eq.(29); the characteristic parameter $\lambda_{n}$ depends on the polynomial's degree ' $n$ ' and is defined by

$$
\lambda_{n}=-n\left(\frac{n-1}{2} A^{\prime \prime}+B^{\prime}\right)
$$

The weight function $\rho(z, \alpha, \beta, n)$ of polynomial solutions is given by

and satisfies the condition

$$
(A \rho)^{\prime}=B \rho,
$$

$$
\lim _{z \rightarrow a} A \rho=\lim _{z \rightarrow b} A \rho=0
$$

where $a$ and $b$ are the end points of the interval of orthogonality of polynomial solutions and $z, \alpha, \beta \in \mathbf{R}$ or $\mathbf{C} ; n \in \mathbf{N}$.

As we can see, Eq.(29) is different from the second-order classical hypergeometric DEs (i-iv) in that-the coefficient of $u^{\prime}$ contains the positive integral parameter ' $n$ ' which is the degree of polynomial 
solutions of Eq.(29). Furthermore, the explicit presence of polynomial's degree ' $n$ ' in the coefficients of $u^{\prime}$ and $u$ implying that the sequences $\{B(z, \alpha, \beta, n)\}_{n=0}^{\infty}$ and $\left\{\lambda_{n}\right\}_{n=0}^{\infty}$ correspond to a unique sequence $\left\{\pi_{n}^{(\alpha, \beta)}(z)\right\}_{n=0}^{\infty}$ of polynomial solutions of Eq.(29).

\section{1. $\pi$-polynomials}

Throughout the rest of this paper we concentrate exclusively on the derivation of $\pi$-polynomials and their DE, generating function, recurrence relation, derivative formula, weight function, Rodrigues' formula, characteristic values as well as their properties . But first, let us begin with their derivation as follows. Assuming $y$ is fixed thus by letting $z=x^{-1}$ and $\lambda=(\alpha+\beta)=y^{-1}$ we get, after substitution in (19):

$$
\frac{1}{n !} Q_{n}(z, \lambda)=\sum_{k=0}^{n} \frac{\lambda^{k}}{k !(n-k) !} z^{n-k}
$$

Now replacing $\lambda^{k}$ by $\lambda^{(k)}=\lambda(\lambda+1)(\lambda+2) \cdots(\lambda+k-1)=\Gamma(\lambda+k) / \Gamma(\lambda), \lambda^{(0)}=1$. We obtain

$$
\frac{1}{n !} Q_{n}(z, \lambda)=\pi_{n}^{(\alpha, \beta)}(z)
$$

where

$$
\pi_{n}^{(\alpha, \beta)}(z)=\sum_{k=0}^{n} \frac{\lambda^{(k)}}{k !(n-k) !} z^{n-k}, \quad \lambda=(\alpha+\beta)
$$

are the very expected $\pi$-polynomials of $\operatorname{bi-order}(\alpha, \beta)$ and degree $n$ in $z$, which satisfy the nonclassical second-order hypergeometric linear DE:

$$
z u^{\prime \prime}-(z+\lambda+n-1) u^{\prime}+n u=0, \lambda=(\alpha+\beta) .
$$

Furthermore, the $\tilde{\pi}$-polynomials defined by the following explicit formula

$$
\tilde{\pi}_{n}^{(\alpha, \beta)}(z)=\sum_{k=0}^{n} \frac{n_{(k)}}{k ! \gamma_{(k)}} z^{k}, \quad \gamma=\lambda+n-1,
$$

are also solutions of Eq.(36).

The first few $\pi$-polynomials

$$
\begin{aligned}
& \pi_{0}^{(\alpha, \beta)}(z)=1 \\
& \pi_{1}^{(\alpha, \beta)}(z)=z+\lambda^{(1)} \\
& \pi_{2}^{(\alpha, \beta)}(z)=\frac{1}{2}\left[z^{2}+2 \lambda^{(1)} z+\lambda^{(2)}\right] \\
& \pi_{3}^{(\alpha, \beta)}(z)=\frac{1}{6}\left[z^{3}+3 \lambda^{(1)} z^{2}+3 \lambda^{(2)} z+\lambda^{(3)}\right] \\
& \pi_{4}^{(\alpha, \beta)}(z)=\frac{1}{24}\left[z^{4}+4 \lambda^{(1)} z^{3}+6 \lambda^{(2)} z^{2}+4 \lambda^{(3)} z+\lambda^{(4)}\right]
\end{aligned}
$$




$$
\begin{aligned}
& \pi_{5}^{(\alpha, \beta)}(z)=\frac{1}{120}\left[z^{5}+5 \lambda^{(1)} z^{4}+10 \lambda^{(2)} z^{3}+10 \lambda^{(3)} z^{2}+5 \lambda^{(4)} z+\lambda^{(5)}\right] \\
& \pi_{6}^{(\alpha, \beta)}(z)=\frac{1}{720}\left[z^{6}+6 \lambda^{(1)} z^{5}+15 \lambda^{(2)} z^{4}+20 \lambda^{(3)} z^{3}+15 \lambda^{(4)} z^{2}+6 \lambda^{(5)} z+\lambda^{(6)}\right]
\end{aligned}
$$

Generating function for $\pi$-polynomials

$$
g(z, t, \alpha, \beta)=\frac{e^{z t}}{(1-t)^{\lambda}}, \lambda=(\alpha+\beta) .
$$

Recurrence relation

$$
(n+1) \pi_{n+1}^{(\alpha, \beta)}(z)=(z+\lambda+n) \pi_{n}^{(\alpha, \beta)}(z)-z \pi_{n-1}^{(\alpha, \beta)}(z) .
$$

Derivative formula

$$
\frac{d \pi_{n}^{(\alpha, \beta)}(z)}{d z}=\pi_{n-1}^{(\alpha, \beta)}(z)
$$

Weight function

$$
\rho(z, \alpha, \beta, n)=z^{-(\lambda+n)} e^{-z}, \lambda=(\alpha+\beta) .
$$

The analogue of Rodrigues' formula

$$
\pi_{n}^{(\alpha, \beta)}(z)=\frac{(-1)^{n}}{n !} z^{\lambda+n} e^{z} \frac{d^{n}}{d z^{n}}\left(z^{-\lambda} e^{-z}\right), \quad \lambda=(\alpha+\beta)
$$

\subsection{Properties of $\pi$-polynomials}

Characteristic values

$$
\begin{aligned}
& \pi_{0}^{(\alpha, \beta)}(z)=1 \\
& \pi_{n}^{(\alpha, \beta)}(0)=\frac{\lambda^{(n)}}{n !}=\frac{\Gamma(\lambda+n)}{n ! \Gamma(\lambda)} \\
& \pi_{n}^{(\alpha, \beta)}(1)=\sum_{k=0}^{n} \frac{\lambda^{(k)}}{k !(n-k) !}=\sum_{k=0}^{n} \frac{\Gamma(\lambda+k)}{k !(n-k) ! \Gamma(\lambda)} \\
& \pi_{n}^{(\alpha, \beta)}(-1)=\sum_{k=0}^{n}(-1)^{(n-k)} \frac{\lambda^{(k)}}{k !(n-k) !}=\sum_{k=0}^{n}(-1)^{(n-k)} \frac{\Gamma(\lambda+k)}{k !(n-k) ! \Gamma(\lambda)} \\
& \pi_{n}^{(1 / 2,1 / 2)}(0)=1 \\
& \pi_{n}^{(-1 / 2,-1 / 2)}(0)=0 \\
& \pi_{n}^{(1 / 2,1 / 2)}(1)=\sum_{k=0}^{n} \frac{1}{(n-k) !} \\
& \pi_{n}^{(-1 / 2,-1 / 2)}(1)=\sum_{k=0}^{n} \frac{(-1)^{(n-k)}}{(n-k) !} .
\end{aligned}
$$




\section{$\pi$-monomial}

We get the $\pi$-monomial for the interesting special case when $\alpha=\beta=0$, that is $\pi_{n}^{(0,0)}(z)=\frac{z^{n}}{n !}$ which satisfies the DE:

$$
z u^{\prime \prime}-(z+n-1) u^{\prime}+n u=0
$$

\section{Orthogonality}

Now we prove the orthogonality of $\pi$-polynomials w.r.t the weight function (42) on the interval $(0, \infty)$. That is to say, we will get $\int_{0}^{\infty} \rho \pi_{m}^{(\alpha, \beta)}(z) \pi_{n}^{(\alpha, \beta)}(z) d z=0$ if $m \neq n$. To this end, let us rewrite Eq.(36) in the following self-adjoint form

$$
\frac{d}{d z}\left(z \rho u^{\prime}\right)+n \rho u=0
$$

Then by Eq. (45) we get

$$
\begin{aligned}
& \frac{d}{d z}\left[z \rho \pi_{m}^{(\alpha, \beta)}(z)\right]+m \rho \pi_{m}^{(\alpha, \beta)}(z)=0, \\
& \frac{d}{d z}\left[z \rho \pi_{n}^{\prime(\alpha, \beta)}(z)\right]+n \rho \pi_{n}^{(\alpha, \beta)}(z)=0 .
\end{aligned}
$$

Multiply (46) by $\pi_{n}^{(\alpha, \beta)}(z)$ and integrate from $z=0$ to $z=\infty$ to obtain

$$
\int_{0}^{\infty} \frac{d}{d z}\left[z \rho \pi_{m}^{(\alpha, \beta)}(z)\right] \pi_{n}^{(\alpha, \beta)}(z) d z+m \int_{0}^{\infty} \rho \pi_{m}^{(\alpha, \beta)}(z) \pi_{n}^{(\alpha, \beta)}(z) d z=0
$$

Integrating the first integral by parts we get

$$
\left[z \rho \pi_{m}^{\prime(\alpha, \beta)}(z) \pi_{n}^{(\alpha, \beta)}(z)\right]_{0}^{\infty}-\int_{0}^{\infty} z \rho \pi_{m}^{\prime(\alpha, \beta)}(z) \pi_{n}^{\prime(\alpha, \beta)}(z) d z+m \int_{0}^{\infty} \rho \pi_{m}^{(\alpha, \beta)}(z) \pi_{n}^{(\alpha, \beta)}(z) d z=0
$$

Since $\lim _{z \rightarrow 0}(z \rho)=\lim _{z \rightarrow 0}\left(z^{1-\gamma} e^{-z}\right)=0$ and $\lim _{z \rightarrow \infty}(z \rho)=\lim _{z \rightarrow \infty}\left(z^{1-\gamma} e^{-z}\right)=0$ with $\gamma=\lambda+n<1$, thus the above expression becomes

$$
-\int_{0}^{\infty} z \rho \pi_{m}^{\prime(\alpha, \beta)}(z) \pi_{n}^{\prime(\alpha, \beta)}(z) d z+m \int_{0}^{\infty} \rho \pi_{m}^{(\alpha, \beta)}(z) \pi_{n}^{(\alpha, \beta)}(z) d z=0
$$

In exactly the same way we can multiply (47) by $\pi_{m}^{(\alpha, \beta)}(z)$ and integrate by parts from $z=0$ to $z=\infty$ to obtain

$$
-\int_{0}^{\infty} z \rho \pi_{m}^{\prime(\alpha, \beta)}(z) \pi_{n}^{\prime(\alpha, \beta)}(z) d z+n \int_{0}^{\infty} \rho \pi_{m}^{(\alpha, \beta)}(z) \pi_{n}^{(\alpha, \beta)}(z) d z=0 .
$$

Finally, subtracting (49) from (48) we get the very expected orthogonality condition

$$
\int_{0}^{\infty} \rho \pi_{m}^{(\alpha, \beta)}(z) \pi_{n}^{(\alpha, \beta)}(z) d z=0, \quad m \neq n
$$


At present, let us evaluate the integral $\int_{0}^{\infty} \rho\left[\pi_{n}^{(\alpha, \beta)}(z)\right]^{2} d z$. That is to say, the integral (50) for the case when $m=n$. First, we have

$$
I_{n}=\int_{0}^{\infty} \rho\left[\pi_{n}^{(\alpha, \beta)}(z)\right]^{2} d z
$$

and

$$
\pi_{n}^{(\alpha, \beta)}=\frac{(-1)^{n}}{n !} \rho^{-1} \frac{d^{n}}{d z^{n}}\left[z^{n} \rho\right]
$$

From (51) and (52) we get

$$
I_{n}=\frac{(-1)^{n}}{n !} \int_{0}^{\infty} \pi_{n}^{(\alpha, \beta)} \frac{d^{n}}{d z^{n}}\left[z^{-\lambda} e^{-z}\right] d z
$$

Integration by parts gives

$$
I_{n}=\left[\frac{(-1)^{n}}{n !} \pi_{n}^{(\alpha, \beta)} \frac{d^{n-1}}{d z^{n-1}}\left(z^{-\lambda} e^{-z}\right)\right]_{0}^{\infty}-\frac{(-1)^{n}}{n !} \int_{0}^{\infty} \frac{d}{d z} \pi_{n}^{(\alpha, \beta)} \frac{d^{n-1}}{d z^{n-1}}\left(z^{-\lambda} e^{-z}\right) d z
$$

The first term on the RHS is zero, hence by integrating several times by parts, we find

$$
I_{n}=\frac{1}{n !} \int_{0}^{\infty} z^{-\lambda} e^{z} \frac{d^{n}}{d z^{n}} \pi_{n}^{(\alpha, \beta)} d z
$$

Since $\frac{d^{n}}{d z^{n}} \pi_{n}^{(\alpha, \beta)}=1$ and $\int_{0}^{\infty} z^{-\lambda} e^{z} d z=\Gamma(1-\lambda)$ for $\lambda<1$ thus the above integral becomes

$$
I_{n}=\frac{\Gamma(1-\lambda)}{n !}
$$

From (51) and (55) we obtain the very expected expression

$$
\int_{0}^{\infty} \rho\left[\pi_{n}^{(\alpha, \beta)}\right]^{2} d z=\frac{\Gamma(1-\lambda)}{n !}, \quad \lambda=\alpha+\beta<1
$$

Finally, combining (50) and (56) we get

$$
\int_{0}^{\infty} \rho \pi_{m}^{(\alpha, \beta)}(z) \pi_{n}^{(\alpha, \beta)}(z) d z=\delta_{m n} \frac{\Gamma(1-\lambda)}{n !}
$$

where $\lambda=\alpha+\beta<1$ and $\delta_{m n}$ is the Kronecker delta defined as $\delta_{m n}=\left\{\begin{array}{l}0 \text { if } m \neq n \\ 1 \text { if } m=n\end{array}\right.$.

\subsection{Series of $\pi$-polynomials}

As a direct consequence of $\pi$-polynomials we can refer to the series of $\pi$-polynomials, that is to say, any continuous function $f(z)$ on the interval $(0, \infty)$ such that $|z|<1$, may be expanded in series of $\pi$ polynomials. More precisely, let us prove that if

$$
f(z)=\sum_{k=0}^{\infty} c_{n} \pi_{n}^{(\alpha, \beta)}(z), \quad|z|<1,
$$


this implies

$$
c_{n}=\frac{n !}{\Gamma(1-\lambda)} \int_{0}^{\infty} \rho \pi_{n}^{(\alpha, \beta)}(z) f(z) d z, \quad \lambda<1
$$

To this end, multiplying the series (58) by $\rho \pi_{m}^{(\alpha, \beta)}(z)$ and integrating from $z=0$ to $z=\infty$ and taking into account the previous result namely the expression (56), we get

$$
\int_{0}^{\infty} \rho \pi_{m}^{(\alpha, \beta)}(z) f(z) d z=\sum_{n=0}^{\infty} c_{n} \int_{0}^{\infty} \rho \pi_{m}^{(\alpha, \beta)}(z) \pi_{n}^{(\alpha, \beta)}(z) d z
$$

For the case when $m=n$, we have

$$
\int_{0}^{\infty} \rho \pi_{n}^{(\alpha, \beta)}(z) f(z) d z=c_{n} \int_{0}^{\infty} \rho\left[\pi_{n}^{(\alpha, \beta)}(z)\right]^{2} d z
$$

from where we can obtain the very expected formula (59).

Illustrative example: Let $z \mapsto f(z)=z^{3}-3 z^{2}+z-2$ be a continuous function on the interval $(0, \infty)$ such that $|z|<1$. Our aim is to find the expansion of $f(z)$ in series of $\pi$-polynomials for the interesting case when $\alpha=\beta=-n / 2$, i.e., $\lambda=-n$. First, we have according to (35):

$$
\pi_{n}^{(-n / 2,-n / 2)}(z)=\frac{1}{n !} \sum_{k=0}^{n}(-1)^{k} \frac{n_{(k)}^{2}}{k !} z^{n-k}=\frac{1}{n !}\left[z^{n}-\frac{n_{(1)}^{2}}{1 !} z^{n-1}+\frac{n_{(2)}^{2}}{2 !} z^{n-2}-\frac{n_{(3)}^{2}}{3 !} z^{n-3}+\cdots+(-1)^{k} n !\right]
$$

The few first $\pi$-polynomials for $\alpha=\beta=-n / 2$

$$
\begin{aligned}
& \pi_{0}^{(0,0)}(z)=1 \\
& \pi_{n}^{(-1 / 2,-1 / 2)}(z)=z-1 \\
& \pi_{2}^{(-1,-1)}(z)=\frac{1}{2}\left(z^{2}-4 z+2\right) \\
& \pi_{3}^{(-3 / 2,-3 / 2)}(z)=\frac{1}{6}\left(z^{3}-9 z^{2}+18 z-6\right) \\
& \pi_{4}^{(-2,-2)}(z)=\frac{1}{24}\left(z^{4}-16 z^{3}+72 z^{2}-96 z+24\right) \\
& \pi_{5}^{(-5 / 2,-5 / 2)}(z)=\frac{1}{120}\left(z^{5}-25 z^{4}+200 z^{3}-600 z^{2}+600 z-120\right) \\
& \pi_{6}^{(-3,-3)}(z)=\frac{1}{720}\left(z^{6}-360 z^{5}+450 z^{4}-2400 z^{3}+5400 z^{2}-4320 z+720\right)
\end{aligned}
$$

We have according to (58)

$$
f(z)=\sum_{k=0}^{\infty} c_{n} \pi_{n}^{(-n / 2,-n / 2)}(z)=c_{0} \pi_{0}^{(0,0)}(z)+c_{1} \pi_{1}^{(-1 / 2,-1 / 2)}(z)+c_{2} \pi_{2}^{(-1,-1)}(z)+c_{3} \pi_{3}^{(-3 / 2,-3 / 2)}(z)+\cdots
$$

It is clear from the explicit expression of $f(z)$, that is, we should have $c_{4}=c_{5}=c_{6}=\cdots=0$. Consequently, the above expression becomes 


$$
f(z)=\sum_{k=0}^{3} c_{n} \pi_{n}^{(-n / 2,-n / 2)}(z)=c_{0} \pi_{0}^{(0,0)}(z)+c_{1} \pi_{1}^{(-1 / 2,-1 / 2)}(z)+c_{2} \pi_{2}^{(-1,-1)}(z)+c_{3} \pi_{3}^{(-3 / 2,-3 / 2)}(z) .
$$

Using the formula (59) to get the following values for the coefficients: $c_{0}=-1, c_{1}=7, c_{2}=12, c_{3}=6$. Finally, after substitution, we obtain the requested expansion:

$$
f(z)=-\pi_{0}^{(0,0)}(z)+7 \pi_{1}^{(-1 / 2,-1 / 2)}(z)+12 \pi_{2}^{(-1,-1)}(z)+6 \pi_{3}^{(-3 / 2,-3 / 2)}(z) .
$$

\subsection{Consequences of $\pi$-polynomials}

The consequences and applications of $\pi$-polynomials are generally related to the specialization of their bi-order $(\alpha, \beta)$. For instance, as a direct consequence of $\pi$-polynomials we can refer to the series of $\pi$ polynomials. It may be added that the $\pi$-polynomials and their reverse can be used in physics particularly in non-relativistic and relativistic quantum mechanics as we will see. With this aim, we shall now consider the following interesting special case, that is, when $\alpha=-a, \beta=-n$ where, as usual, $a \in \mathbf{R}$ or $\mathbf{C}$ and $n \in \mathbf{N}$. Therefore, after substitution, the $\pi$-polynomials (35) and their DE (36) become

$$
\begin{gathered}
\pi_{n}^{(-a,-n)}(z)=\sum_{k=0}^{n}(-1)^{k}\left(\begin{array}{c}
a+n \\
k
\end{array}\right) \frac{z^{n-k}}{(n-k) !}, \\
z u^{\prime \prime}+(a+1-z) u^{\prime}+n u=0 .
\end{gathered}
$$

The first few $\pi$-polynomials for $\alpha=-a, \beta=-n$

$$
\begin{aligned}
& \pi_{0}^{(-a, 0)}(z)=1 \\
& \pi_{1}^{(-a,-1)}(z)=z-(a+1)_{(1)} \\
& \pi_{2}^{(-a,-2)}(z)=\frac{z^{2}}{2}-(a+2)_{(1)} z+\frac{(a+2)_{(2)}}{2} \\
& \pi_{3}^{(-a,-3)}(z)=\frac{z^{3}}{6}-\frac{(a+3)_{(1)}}{2} z^{2}+\frac{(a+3)_{(2)}}{2} z-\frac{(a+3)_{(3)}}{6} \\
& \pi_{4}^{(-a,-4)}(z)=\frac{z^{4}}{24}-\frac{(a+4)_{(1)}}{6} z^{3}+\frac{(a+4)_{(2)}}{4} z^{2}-\frac{(a+4)_{(3)}}{6} z+\frac{(a+4)_{(4)}}{24} \\
& \pi_{5}^{(-a,-5)}(z)=\frac{z^{5}}{120}-\frac{(a+5)_{(1)}}{24} z^{4}+\frac{(a+5)_{(2)}}{12} z^{3}-\frac{(a+5)_{(3)}}{12} z^{2}+\frac{(a+5)_{(4)}}{24} z-\frac{(a+5)_{(5)}}{120}
\end{aligned}
$$

It is worthwhile to note that in spite of the fact that Eq.(63) is identical to the so-called Laguerre (generalized) equation ${ }^{(2)}$, the polynomials (62) are not strictly speaking identical to the alleged generalized (associated) Laguerre polynomials

(2) The so-called generalized Laguerre equation and polynomials have been wrongly attributed to the French mathematician Edmond Nicolas Laguerre (1834-1886) by Encyclopedia of Mathematics, Encyclopedic Dictionary of Mathematics, Wikipedia, Wolfram MathWorld, and also by many authors of mathematics textbooks and peer-reviewed research articles relating to the classical orthogonal polynomials. Laguerre had nothing to do with such a wrong attribution and the actual discoverer was the Russian mathematician Nikolay Yacovlevich Sonine (1849-1915), see Ref.[13]. -Laguerre true and 


$$
L_{n}^{(a)}(z)=\sum_{k=0}^{n}(-1)^{k}\left(\begin{array}{c}
a+n \\
n-k
\end{array}\right) \frac{z^{k}}{k !}
$$

Since both polynomials (62) and (i) have not the same explicit expressions for the recurrence relations and derivative formulae, respectively:

$$
(n+1) \pi_{n+1}^{(-a,-n)}(z)=(a+n+1-z) \pi_{n}^{(-a,-n)}(z)-z \pi_{n-1}^{(-a,-n)}(z) ; \quad \frac{d \pi_{n}^{(-a,-n)}(z)}{d z}=\pi_{n-1}^{(-a,-n)}(z)-\frac{d \pi_{n-1}^{(-a,-n)}(z)}{d z}
$$

and

$$
(n+1) L_{n+1}^{(a)}(z)=(a+2 n+1-z) L_{n}^{(a)}(z)-(a+n) L_{n-1}^{(a)}(z) ; \frac{d L_{n}^{(a)}(z)}{d z}=-L_{n-1}^{(a+1)}(z) .
$$

This implies, among other things, that they have not the same generating function. Consequently, the polynomials $\pi_{n}^{(-a,-n)}(z)$ can also be used in non-relativistic and relativistic QM, particularly, in the case of the Schrödinger equation, and Dirac equations for an electron in a Coulomb potential field. However, we can show that there is some link between $\pi_{n}^{(-a,-n)}(z)$ and $L_{n}^{(a)}(z)$ through the reverse polynomials $\bar{\pi}_{n}^{(-a,-n)}(z)$, which can be easily deduced from (62):

$$
\bar{\pi}_{n}^{(-a,-n)}(z)=\sum_{k=0}^{n}(-1)^{n-k}\left(\begin{array}{c}
a+n \\
n-k
\end{array}\right) \frac{z^{k}}{k !} .
$$

The first six $\bar{\pi}$-polynomials

$$
\begin{aligned}
& \bar{\pi}_{0}^{(-a, 0)}(z)=1 \\
& \bar{\pi}_{1}^{(-a,-1)}(z)=-(a+1)_{(1)}+z \\
& \bar{\pi}_{2}^{(-a,-2)}(z)=\frac{(a+2)_{(2)}}{2}-(a+2)_{(1)} z+\frac{z^{2}}{2} \\
& \bar{\pi}_{3}^{(-a,-3)}(z)=-\frac{(a+3)_{(3)}}{6}+\frac{(a+3)_{(2)}}{2} z-\frac{(a+3)_{(1)}}{2} z^{2}+\frac{z^{3}}{6} \\
& \bar{\pi}_{4}^{(-a,-4)}(z)=\frac{(a+4)_{(4)}}{24}-\frac{(a+4)_{(3)}}{6} z+\frac{(a+4)_{(2)}}{4} z^{2}-\frac{(a+4)_{(1)}}{6} z^{3}+\frac{z^{4}}{24} \\
& \bar{\pi}_{5}^{(-a,-5)}(z)=-\frac{(a+5)_{(5)}}{120}+\frac{(a+5)_{(4)}}{24} z-\frac{(a+5)_{(3)}}{12} z^{2}+\frac{(a+5)_{(2)}}{12} z^{3}-\frac{(a+5)_{(1)}}{24} z^{4}+\frac{z^{5}}{120} .
\end{aligned}
$$

As we can see from (64) and (66), the polynomials $\pi_{n}^{(-a,-n)}(z)$ are arranged in descending power order of $z$ while the polynomials $\bar{\pi}_{n}^{(-a,-n)}(z)$ are arranged in ascending power order of $z$, and as the descending power order is more commonly used that is why (65) are called the 'reverse' polynomials or simply $\bar{\pi}$. polynomials.

authentic ODEs and polynomials: Laguerre published two seminal articles, respectively, in 1879 Ref.[14] in which he explicitly wrote the equation: $x y^{\prime \prime}+(1+x) y^{\prime}-n y=0$ and its polynomial solution; and in his second article published in 1885 Ref.[15] in which he explicitly wrote the equation: $x y^{\prime \prime}+(x+1-\alpha) y^{\prime}-n y=0$ and its polynomial solution. As we can see more clearly, the first Eq. is a special case of the second Eq. when $\alpha=0$ and both Eqs. are different from $x y^{\prime \prime}+(1-x) y^{\prime}+n y=0$ and $x y^{\prime \prime}+(\alpha+1-x) y^{\prime}+n y=0$, which are wrongly attributed to Laguerre. 
We now proceed to show that there is actually some link between $\pi_{n}^{(-a,-n)}(z)$ and $L_{n}^{(a)}(z)$ through $\bar{\pi}_{n}^{(-a,-n)}(z)$, which can be rewritten in the following form:

$$
\bar{\pi}_{n}^{(-a,-n)}(z)=(-1)^{n} \sum_{k=0}^{n}(-1)^{k}\left(\begin{array}{l}
a+n \\
n-k
\end{array}\right) \frac{z^{k}}{k !}
$$

a simple comparison between (i) and (67) leads directly to the expression:

$$
\bar{\pi}_{n}^{(-a,-n)}(z)=(-1)^{n} L_{n}^{(a)}(z),
$$

or equivalently

$$
L_{n}^{(a)}(z)=(-1)^{n} \bar{\pi}_{n}^{(-a,-n)}(z)
$$

Expressions (68) and (69) mean that the polynomials $\bar{\pi}_{n}^{(-a,-n)}(z)$ are expressible in terms of the polynomials $L_{n}^{(a)}(z)$ and vice versa. Moreover, the polynomials $\pi_{n}^{(-a,-n)}(z)$ are related to the Hermite ones through $\bar{\pi}_{n}^{(-a,-n)}(z)$ :

$$
\begin{gathered}
H_{2 n}(z)=2^{2 n} n ! \bar{\pi}_{n}^{(1 / 2,-n)}\left(z^{2}\right), \\
H_{2 n+1}(z)=2^{2 n+1} n ! z \bar{\pi}_{n}^{(-1 / 2,-n)}\left(z^{2}\right) .
\end{gathered}
$$

Because of this, the polynomials $\bar{\pi}_{n}^{(-a,-n)}(z)$ can be arisen in the treatment of the quantum harmonic oscillator.

\section{Associated $\varphi$-functions}

In terms of the polynomials $\pi_{n}^{(-a,-n)}(z)$, we can define the associated $\varphi$-functions as:

$$
\varphi_{n}(z, a)=z^{\frac{a}{2}} e^{-\frac{z}{2}} \pi_{n}^{(-a,-n)}(z)
$$

Using Eq.(63), we get the following self-adjoint DE for $\varphi$-functions

$$
z \varphi_{n}^{\prime \prime}+\varphi_{n}^{\prime}+\left[\frac{2 n+a+1}{2}-\frac{z}{4}-\frac{a^{2}}{4 z}\right] \varphi_{n}=0 .
$$

Therefore, $\varphi$-functions are eigen-functions of a Sturm-Liouville system on the interval $(0, \infty)$.

\section{$\tilde{\pi}$-polynomials}

Now returning to the $\tilde{\pi}$-polynomials (37), solutions of Eq.(36), and considering the same previous special case, i.e., when $\alpha=-a, \beta=-n$, we get after substitution in (37):

$$
\tilde{\pi}_{n}^{(-a,-n)}(z)=\sum_{k=0}^{n}(-1)^{k} \frac{n_{(k)}}{(a+1)^{(k)}} \frac{z^{k}}{k !},
$$

or equivalently 


$$
\tilde{\pi}_{n}^{(-a,-n)}(z)=\frac{1}{(a+1)^{(n)}} \sum_{k=0}^{n}(-1)^{k} \frac{n_{(k)}}{k !}(a+n)_{(n-k)} z^{k}
$$

which are also solutions of Eq.(63).

The first six $\tilde{\pi}$-polynomials

$\tilde{\pi}_{0}^{(-a, 0)}(z)=1$

$\tilde{\pi}_{1}^{(-a,-1)}(z)=1-\frac{1}{(a+1)^{(1)}} z$

$\tilde{\pi}_{2}^{(-a,-2)}(z)=1-\frac{2}{(a+1)^{(1)}} z+\frac{1}{(a+1)^{(2)}} z^{2}$

$\tilde{\pi}_{3}^{(-a,-3)}(z)=1-\frac{3}{(a+1)^{(1)}} z+\frac{3}{(a+1)^{(2)}} z^{2}-\frac{1}{(a+1)^{(3)}} z^{3}$

$\tilde{\pi}_{4}^{(-a,-4)}(z)=1-\frac{4}{(a+1)^{(1)}} z+\frac{6}{(a+1)^{(2)}} z^{2}-\frac{4}{(a+1)^{(3)}} z^{3}+\frac{1}{(a+1)^{(4)}} z^{4}$

$\tilde{\pi}_{5}^{(-a,-5)}(z)=1-\frac{5}{(a+1)^{(1)}} z+\frac{10}{(a+1)^{(2)}} z^{2}-\frac{10}{(a+1)^{(3)}} z^{3}+\frac{5}{(a+1)^{(4)}} z^{4}-\frac{1}{(a+1)^{(5)}} z^{5}$.

The analogue of Ridrogues' formula

$$
\tilde{\pi}_{n}^{(-a,-n)}(z)=\frac{1}{(a+n)_{(n)}} z^{-a} e^{z} \frac{d^{n}}{d z^{n}}\left(z^{a+n} e^{-z}\right) .
$$

The polynomials (74) are related to $\pi_{n}^{(-a,-n)}(z)$ for $\alpha=-a$ and $\beta=-n$ by the following relation:

$$
\pi_{n}^{(-a,-n)}(z)=(-1)^{n} \frac{(a+n)_{(n)}}{n !} \tilde{\boldsymbol{\pi}}_{n}^{(-a,-n)}(z)
$$

It may be added that the orthogonality property of the polynomials (74) is a direct consequence of the orthogonality of $\pi$-polynomials (35) and without difficulty can be shown to be of the form:

$$
\int_{0}^{\infty} z^{a} e^{-z} \tilde{\pi}_{m}^{(-a,-n)}(z) \tilde{\pi}_{n}^{(-a,-n)}(z) d z=\delta_{m n} \frac{n ![\Gamma(a+1)]^{2}}{\Gamma(a+n+1)}
$$

\section{5. $\mu$-monomials and $\kappa$-polynomials}

In addition to the properties of $\pi$-polynomials (35) mentioned in the previous section, there is another specific property which is in fact a direct consequence of the explicit expression of $\pi$-polynomials which can also be written in the form of the product of $\mu$-monomials in $z$ and $\kappa$-polynomials in $\left(z^{-1}\right)$ as follows:

$$
\pi_{n}^{(\alpha, \beta)}(z)=\sum_{k=0}^{n} \frac{\lambda^{(k)}}{k !(n-k) !} z^{n-k}=z^{n} \sum_{k=0}^{n} \frac{\lambda^{(k)}}{k !(n-k) !} \frac{1}{z^{k}}=\mu_{n}(z) \boldsymbol{\kappa}_{n}^{(\alpha, \beta)}(z)
$$


where the $\mu$-monomials $\mu_{n}(z)=z^{n}$ or equivalently $\mu_{n}(z)=n ! \pi_{n}^{(0,0)}(z)$ satisfy Eq.(44).

Now, let us focus our attention exclusively on the $\kappa$-polynomials

which satisfy the DE

$$
\kappa_{n}^{(\alpha, \beta)}(z)=\sum_{k=0}^{n} \frac{\lambda^{(k)}}{k !(n-k) !} \frac{1}{z^{k}}, \lambda=\alpha+\beta
$$

$$
z^{2} w^{\prime \prime}+z(n-\lambda+1-z) w^{\prime}-n \lambda w=0
$$

The first few K-polynomials

$$
\begin{aligned}
& \boldsymbol{K}_{0}^{(\alpha, \beta)}(z)=1 \\
& \boldsymbol{K}_{1}^{(\alpha, \beta)}(z)=1+\lambda z^{-1} \\
& \boldsymbol{K}_{2}^{(\alpha, \beta)}(z)=\frac{1}{2}+\lambda z^{-1}+\frac{\lambda^{(2)}}{2} z^{-2} \\
& \boldsymbol{K}_{3}^{(\alpha, \beta)}(z)=\frac{1}{6}+\frac{\lambda}{2} z^{-1}+\frac{\lambda^{(2)}}{2} z^{-2}+\frac{\lambda^{(3)}}{6} z^{-3} \\
& \boldsymbol{K}_{4}^{(\alpha, \beta)}(z)=\frac{1}{24}+\frac{\lambda}{6} z^{-1}+\frac{\lambda^{(2)}}{4} z^{-2}+\frac{\lambda^{(3)}}{6} z^{-3}+\frac{\lambda^{(4)}}{24} z^{-4} \\
& \boldsymbol{K}_{5}^{(\alpha, \beta)}(z)=\frac{1}{120}+\frac{\lambda}{24} z^{-1}+\frac{\lambda^{(2)}}{12} z^{-2}+\frac{\lambda^{(3)}}{12} z^{-3}+\frac{\lambda^{(4)}}{24} z^{-4}+\frac{\lambda^{(5)}}{120} z^{-5} \\
& \boldsymbol{K}_{6}^{(\alpha, \beta)}(z)=\frac{1}{720}+\frac{\lambda}{120} z^{-1}+\frac{\lambda^{(2)}}{48} z^{-2}+\frac{\lambda^{(3)}}{36} z^{-3}+\frac{\lambda^{(4)}}{48} z^{-4}+\frac{\lambda^{(5)}}{120} z^{-5}+\frac{\lambda^{(6)}}{720} z^{-6}
\end{aligned}
$$

Generating function for K-polynomials

Recurrence relation

$$
g(z, t, \alpha, \beta)=\left(1-\frac{t}{z}\right)^{-\lambda} e^{t}, \quad \lambda=\alpha+\beta
$$

$$
(n+1) \boldsymbol{\kappa}_{n+1}^{(\alpha, \beta)}(z)=\left(1+\frac{\lambda+n}{z}\right) \boldsymbol{\kappa}_{n}^{(\alpha, \beta)}(z)-\frac{\lambda}{z^{2}} \boldsymbol{\kappa}_{n-1}^{(\alpha, \beta)}(z) .
$$

Derivative formula

Weight function

$$
\frac{d \boldsymbol{\kappa}_{n}^{(\alpha, \beta)}(z)}{d z}=\frac{1}{z} \frac{d \boldsymbol{\kappa}_{n-1}^{(\alpha, \beta)}(z)}{d z}-\frac{\lambda}{z^{2}} \boldsymbol{\kappa}_{n-1}^{(\alpha, \beta)}(z)
$$

$$
\rho(z, \alpha, \beta, n)=z^{\gamma} e^{-z}, \quad \gamma=n-\lambda-1 .
$$

The analogue of Rodrigues' formula

$$
\boldsymbol{\kappa}_{n}^{(\alpha, \beta)}(z)=\frac{(-1)^{n}}{n !} z^{\lambda} e^{z} \frac{d^{n}}{d z^{n}}\left[z^{-\lambda} e^{-z}\right]
$$


Characteristic values

$\boldsymbol{K}_{n}^{(\alpha, \beta)}(\infty)=\boldsymbol{K}_{n}^{(0,0)}(z)=\frac{1}{n !}$

$\boldsymbol{K}_{n}^{(\alpha, \beta)}(0)=\infty$

$\kappa_{n}^{(\alpha, \beta)}(1)=\sum_{k=0}^{n} \frac{\lambda^{(k)}}{k !(n-k) !}=\sum_{k=0}^{n} \frac{\Gamma(\lambda+k)}{k !(n-k) ! \Gamma(\lambda)}$

$\kappa_{n}^{(\alpha, \beta)}(-1)=\sum_{k=0}^{n}(-1)^{k} \frac{\lambda^{(k)}}{k !(n-k) !}=\sum_{k=0}^{n}(-1)^{k} \frac{\Gamma(\lambda+k)}{k !(n-k) ! \Gamma(\lambda)}$.

Some properties

$\kappa_{n}^{(\alpha, \beta)}(-z)=\sum_{k=0}^{n}(-1)^{k} \frac{\lambda^{(k)}}{k !(n-k) !} \frac{1}{z^{k}}, \lambda=\alpha+\beta$

$\boldsymbol{K}_{n}^{(-\alpha,-\beta)}(z)=\sum_{k=0}^{n}(-1)^{k} \frac{\lambda_{(k)}}{k !(n-k) !} \frac{1}{z^{k}}$

$\mathcal{K}_{n}^{(-\alpha,-\beta)}(-z)=\sum_{k=0}^{n} \frac{\lambda_{(k)}}{k !(n-k) !} \frac{1}{z^{k}}$

$\mathcal{K}_{n}^{(-\alpha, \beta)}(z)=\sum_{k=0}^{n} \frac{(\beta-\alpha)^{(k)}}{k !(n-k) !} \frac{1}{z^{k}}$

$\boldsymbol{K}_{n}^{(\alpha,-\beta)}(z)=\sum_{k=0}^{n}(-1)^{k} \frac{(\beta-\alpha)_{(k)}}{k !(n-k) !} \frac{1}{z^{k}}$

$\kappa_{n}^{(-\alpha, \beta)}(-z)=\sum_{k=0}^{n}(-1)^{k} \frac{(\beta-\alpha)^{(k)}}{k !(n-k) !} \frac{1}{z^{k}}$

$\boldsymbol{\kappa}_{n}^{(\alpha,-\beta)}(-z)=\sum_{k=0}^{n} \frac{(\beta-\alpha)_{(k)}}{k !(n-k) !} \frac{1}{z^{k}}$.

\section{Orthogonality}

To show the orthogonality property of $\mathcal{K}$-polynomials on the interval $(0, \infty)$ w.r.t the weight function (86) it suffices to follow the usual procedure. With this aim, let us first rewrite Eq.(81) in self-adjoint form.

$$
\frac{d}{d z}\left(z^{2} \rho w^{\prime}\right)-n \lambda \rho w=0
$$

Then by (88) we have

$$
\frac{d}{d z}\left(z^{2} \rho \frac{d \kappa_{m}^{(\alpha, \beta)}(z)}{d z}\right)-m \lambda \rho \mathcal{K}_{m}^{(\alpha, \beta)}(z)=0
$$




$$
\frac{d}{d z}\left(z^{2} \rho \frac{d \kappa_{n}^{(\alpha, \beta)}(z)}{d z}\right)-n \lambda \rho K_{n}^{(\alpha, \beta)}(z)=0
$$

where $m \neq n$.

Now, we multiply (89) by $\kappa_{n}^{(\alpha, \beta)}(z)$ and integrate from $z=0$ to $z=\infty$ to obtain

$$
\int_{0}^{\infty} \frac{d}{d z}\left(z^{2} \rho \frac{d \mathcal{K}_{m}^{(\alpha, \beta)}(z)}{d z}\right) \boldsymbol{\kappa}_{n}^{(\alpha, \beta)}(z) d z-m \lambda \int_{0}^{\infty} \rho \boldsymbol{\kappa}_{m}^{(\alpha, \beta)}(z) \mathcal{K}_{n}^{(\alpha, \beta)}(z) d z=0
$$

Integrating the first integral by parts we get:

$$
\left[z^{2} \rho \frac{d \boldsymbol{\kappa}_{m}^{(\alpha, \beta)}(z)}{d z} \kappa_{n}^{(\alpha, \beta)}(z)\right]_{0}^{\infty}-\int_{0}^{\infty} z^{2} \rho \frac{d \kappa_{m}^{(\alpha, \beta)}(z)}{d z} \frac{d \kappa_{n}^{(\alpha, \beta)}(z)}{d z} d z-m \lambda \int_{0}^{\infty} \rho \boldsymbol{\kappa}_{m}^{(\alpha, \beta)}(z) \boldsymbol{\kappa}_{n}^{(\alpha, \beta)}(z) d z=0
$$

By taking into account the explicit expression of the weight function (86), we have $\lim _{z \rightarrow 0}\left(z^{\gamma+2} e^{-z}\right)=\lim _{z \rightarrow \infty}\left(z^{\gamma+2} e^{-z}\right)=0$ with $\gamma>-2$, thus the above expression becomes:

$$
\int_{0}^{\infty} z^{2} \rho \frac{d \kappa_{m}^{(\alpha, \beta)}(z)}{d z} \frac{d \kappa_{n}^{(\alpha, \beta)}(z)}{d z} d z+m \lambda \int_{0}^{\infty} \rho \mathcal{K}_{m}^{(\alpha, \beta)}(z) \mathcal{K}_{n}^{(\alpha, \beta)}(z) d z=0
$$

In exactly the same way we can multiply $(90)$ by $\kappa_{m}^{(\alpha, \beta)}(z)$ and integrate from $z=0$ to $z=\infty$ to find

$$
\int_{0}^{\infty} z^{2} \rho \frac{d \boldsymbol{\kappa}_{m}^{(\alpha, \beta)}(z)}{d z} \frac{d \kappa_{n}^{(\alpha, \beta)}(z)}{d z} d z+n \lambda \int_{0}^{\infty} \rho \boldsymbol{\kappa}_{m}^{(\alpha, \beta)}(z) \kappa_{n}^{(\alpha, \beta)}(z) d z=0
$$

Subtracting (92) from (91) and replacing the weight function $\rho$ by its explicit expression (86) we get the very expected orthogonality condition:

$$
\int_{0}^{\infty} z^{\gamma} e^{-z} \kappa_{m}^{(\alpha, \beta)}(z) \kappa_{n}^{(\alpha, \beta)}(z) d z=0, \quad m \neq n
$$

Finally, let us evaluate the integral $\int_{0}^{\infty} \rho\left[\boldsymbol{K}_{n}^{(\alpha, \beta)}(z)\right]^{2} d z$ as follows. First, we have

$$
I_{n}=\int_{0}^{\infty} \rho\left[\boldsymbol{K}_{n}^{(\alpha, \beta)}(z)\right]^{2} d z
$$

Secondly, rewriting the generating function in its explicit form

$$
e^{t}\left(1-\frac{t}{z}\right)^{-\lambda}=\sum_{n=0}^{\infty} \boldsymbol{K}_{n}^{(\alpha, \beta)}(z) t^{n}, \quad \lambda=\alpha+\beta
$$

Square and multiply both sides of (95) by the weight function (86) to obtain: 


$$
e^{2 t}\left(1-\frac{t}{z}\right)^{-2 \lambda} \rho=\sum_{n=0}^{\infty} \rho\left[\boldsymbol{K}_{n}^{(\alpha, \beta)}(z)\right]^{2} t^{2 n}
$$

Now, let us focus our attention on the LHS of (96) which can also be written in terms of series as follows:

$$
e^{2 t}\left(1-\frac{t}{z}\right)^{-2 \lambda} \rho=\sum_{n=0}^{\infty} \frac{2^{n}(2 \lambda)^{(n)}}{(n !)^{2}} \frac{t^{2 n}}{z^{n}} \rho=\sum_{n=0}^{\infty} \frac{2^{n}(2 \lambda)^{(n)}}{(n !)^{2}} z^{-(\lambda+1)} e^{-z} t^{2 n}
$$

and after substitution, (96) becomes

$$
\sum_{n=0}^{\infty} z^{\gamma} e^{-z}\left[K_{n}^{(\alpha, \beta)}(z)\right]^{2} t^{2 n}=\sum_{n=0}^{\infty} \frac{2^{n}(2 \lambda)^{(n)}}{(n !)^{2}} z^{-(\lambda+1)} e^{-z} t^{2 n}
$$

with

$$
\gamma=n-\lambda-1, \quad \lambda=\alpha+\beta
$$

Integrating (97) from $z=0$ to $z=\infty$, we get after simplification

$$
\int_{0}^{\infty} z^{\gamma} e^{-z}\left[K_{n}^{(\alpha, \beta)}(z)\right]^{2} d z=\frac{2^{n}(2 \lambda)^{(n)}}{(n !)^{2}} \int_{0}^{\infty} z^{-(\lambda+1)} e^{-z} d z
$$

Since $(2 \lambda)^{(n)}=\Gamma(2 \lambda+n) / \Gamma(2 \lambda)$ and $\int_{0}^{\infty} z^{-(\lambda+1)} e^{-z} d z=\Gamma(-\lambda), \quad \lambda<0$, therefore (98) becomes:

$$
\int_{0}^{\infty} z^{\gamma} e^{-z}\left[\kappa_{n}^{(\alpha, \beta)}(z)\right]^{2} d z=\frac{2^{n} \Gamma(2 \lambda+n)}{(n !)^{2} \Gamma(2 \lambda)} \Gamma(-\lambda)
$$

\section{Conclusion}

In this paper, the usual factorials and binomial coefficients have been generalized and extended by means of the new concept of factorials and combinatorial numbers to the negative integers. Basing on this generalization and extension, a new kind of polynomials has been proposed, which led directly to the non-classical hypergeometric orthogonal polynomials and the non-classical second-order hypergeometric linear DEs. Also the resulting polynomials can be used in non-relativistic and relativistic QM, particularly, in the case of the Schrödinger equation, and Dirac equations for an electron in a Coulomb potential field.

\section{References}

[1] Graham, R. L., Knuth, D. E., Patashnik, O.: Concrete Mathematics. Reading, MA: Addison-Wesley (1988)

[2] Dutka, J.: The early history of factorial function, Arch Hist Exact Sci 43, 225-249 (1991)

[3] Biggs, N. L.: The roots of combinatorics. Historia Mathematica. 6, 109-136 (1979)

[4] Stedman, F.: Campanalogia. London (1677) -The publisher is given as "W.S." who may have been William Smith 
[5] Higgins, P.: Number Story: From Counting to Cryptography. New York: Copernicus (2008)

[6] Gronau, D.: Why is the gamma function so as it is? Teach Math Comput Sci 1, 43-53 (2003)

Srinivasan, G. K.: The gamma function: An eclectic tour. Amer Math Month 114, 297-315 (2007)

[7] Davis, P. J.: Leonhard Euler's Integral: A Historical Profile of the Gamma Function. In Memoriam: Milton Abramowitz. Amer. Math. Monthly 66, 849-869 (1959)

[8] Roman, S.: The logarithmic binomial formula, Am. Math. Month 99, 641-648 (1992)

[9] Bhargava, M.: The Factorial Function and Generalizations. Am. Math. Month 107, 783-799 (2000)

[10] Anglani, R., Barlie, M.: Factorials as Sums. arXiv:Math/0702010v1 (2007)

[11] brahim, A. M.: Extension of factorial concept to negative numbers. Notes Theory Discrete Math 19, 30-42 (2013)

[12] Thukral, A. K.: Factorials of real negative and imaginary numbers - A new perspective. SpringerPlus 3: 658 (2014)

[13] Sonine, N. J.: Sur les fonctions cylindriques et le développement des fonctions continues en séries. Math. Ann. 16, 1-80 (1880)

[14] Laguerre, E.: Sur l'intégrale $\int_{x}^{\infty} x^{-1} e^{-x} d x$. Bull. Soc. math. France 7, 72-81 (1879)

[15] Laguerre, E.: Sur la réduction des fractions continues d'une fraction qui satisfait à une équation différentielle linéaire du premier ordre dont les coefficients sont rationnels. JMPA 4,135-16 (1885)

[16] Chihara, T. S.: An introduction to orthogonal polynomials. Gordon and Breach, New York (1978)

[17] Szegö, G.: Orthogonal polynomials. Amer. Math. Soc. Colloq. Publ. Vol. 23, 4th Edition (1975)

[18] Law, A. G., Sledd, M. B.: A non-classical, orthogonal polynomial family. Lecture Notes in Mathematics, vol.1171. Springer, Berlin, Heidelberg (1985)

[19] Krall, H. L., Frink, O.: A New Class of Orthogonal Polynomials: The Bessel Polynomials. Trans. Amer. Math. Soc. 65, 100-115 (1948) 